\title{
Research on Electrical Equipment Monitoring and Early Warning System Based on Internet of Things Technology
}

\author{
Tianxiang Lei $\mathbb{D},{ }^{1,2}$ Fangcheng Lv, ${ }^{1,2}$ Jiaomin Liu, ${ }^{1}$ and Jiahao Feng ${ }^{3}$ \\ ${ }^{1}$ State Key Laboratory of Alternate Electrical Power System with Renewable Energy Sources, \\ North China Electric Power University, Baoding 071003, China \\ ${ }^{2}$ Hebei Provincial Key Laboratory of Power Transmission Equipment Security Defence, North China Electric Power University, \\ Baoding 071003, China \\ ${ }^{3}$ State Grid Shijiazhuang Electric Power Supply Company, Shijiazhuang 050000, China
}

Correspondence should be addressed to Tianxiang Lei; 1182101032@ncepu.edu.cn

Received 12 January 2022; Accepted 28 January 2022; Published 27 February 2022

Academic Editor: Gengxin Sun

Copyright ( 2022 Tianxiang Lei et al. This is an open access article distributed under the Creative Commons Attribution License, which permits unrestricted use, distribution, and reproduction in any medium, provided the original work is properly cited.

\begin{abstract}
With the rapid increase of global population, the power load increases rapidly, and the electrical equipment such as generator, transformer, and power line in the power system is an important basis for production and life. Its safe operation is of great significance because it is related to people's economic development and life stability. In recent years, with the rapid development of Internet of Things technology, the Internet of Things and electrical equipment safety early warning are combined to use their respective advantages to provide a new way for electrical equipment monitoring and early warning system. Through a variety of key technologies of the Internet of Things, such as sensor technology, network communication technology, and cloud computing technology, data, information exchange, and communication between electrical equipment and Internet technology are carried out according to the agreed protocol, and the safety of the operation status of electrical equipment is monitored in real time, so as to prevent power equipment failure and other problems. Based on this background, this paper studies the Internet of Things technology and electrical equipment monitoring and early warning system and analyzes its three-tier network architecture mode from the Internet of Things technology, namely, perception layer, network layer, and application layer. The combination of cloud computing and edge computing is studied and analyzed to provide theoretical support for the research of electrical equipment monitoring and safety early warning system. The wireless sensor network equipment is also installed on the electrical equipment through the Internet of Things technology to transmit data to the base station, so as to monitor whether the equipment operates safely. The monitoring and early warning system of wireless sensor system based on Internet of Things is given through case experiment. This system realizes relevant intelligent application services, which can not only ensure the stability of information transportation but also real-time monitoring of electrical equipment, early warning, shorten troubleshooting time, reduce the workload of power station staff, and achieve the functions of safety early warning, emergency command, and control. It is of great significance to the monitoring and early warning system of electrical equipment.
\end{abstract}

\section{Introduction}

Nowadays, electric energy is very important to human beings. Now, most power systems adopt unmanned monitoring mode, so the safety of electrical equipment operation is very important. It plays a great role to put data in staff's mobile devices anytime and anywhere through Internet of Things technology.
With the rapid development of Internet technology [1], the Internet of Things technology [2] derived from it has been developed and applied in power, agriculture, environment, and other industries and achieved remarkable results. Especially in the power industry, power [3], as a clean energy for sustainable development [4], has become the main energy for global economic growth at this stage. Therefore, the development and research of its smart grid [5] 
has become a key research object at present. The smart grid constructed by applying Internet of Things technology to power system [6] and intelligent sensing technology [7] has the characteristics of more stable, faster, and more effective information transmission [8].

For the running electrical equipment [9], its temperature is a very important reference base. Its operating temperature, relative temperature rise, and periodic relative temperature difference [10] can reflect whether the equipment is running safely. Any too large or too small temperature may cause irreparable consequences. The equipment is overloaded under high voltage for a long time [11]. At present, most of the electrical equipment adopts the traditional closed structure, and the heat dissipation effect is not obvious, which is more likely to lead to the abnormal increase of its temperature. If it cannot be found and repaired in time, it will cause electrical equipment damage and even more serious power production accidents.

In the condition monitoring mode of electrical equipment based on Internet of Things technology, sensor monitoring network [12] adopts intelligent sensing technology [13], such as FBG sensor [14] and piezoelectric sensor [15] and location tracking technology [16], such as RFID technology [17] or WSN technology [18], for seamless integrated development [19]. Connect the electrical equipment with the Internet, create an intelligent cycle of safety monitoring, prediction, and control [20], and carry out intelligent identification, tracking, and monitoring. Digital wireless temperature monitoring [21] and other equipment are mainly used for real-time monitoring of electrical equipment, power cables, connectors, capacitors, reactors, and other equipment, abandoning the regular maintenance and traditional temperature measurement methods under the traditional mode, such as temperature indicator measurement [22] and infrared temperature measurement [23], monitoring the operation data of electrical equipment in real time through Internet of Things technology and intelligent technology, and evaluating the operation status of equipment, so as to provide decision-making basis for electrical equipment maintenance and ensure its safety and stability. Such a monitoring and early warning system not only saves a lot of human and material resources but also has the characteristics of fast response, high precision, real-time online early warning, rapid fault location, no omission, and strong anti-interference ability. Therefore, the study of electrical equipment monitoring and early warning system on the Internet of Things is of great significance for people's production and life. Intelligent system [24], efficient management, and comfortable and fast electrical equipment monitoring will also bring significant economic benefits to the energy system. The application and development of Internet of Things technology in power system also has a great impact.

\section{Application and Development of Internet of Things Technology in Power System}

2.1. Development of Internet of Things. The concept of the Internet of Things was first put forward by Bill Gates in 1995.
He repeatedly put forward the idea of "Internet of Things." Based on this, after formal and in-depth research by many scientists, the concept of the Internet of Things was obtained, that is, a new world of perception, interconnection, and intelligence of all things. So far, Internet of Things technology has become a major development strategy of countries all over the world. Figure 1 briefly describes the development background of the Internet of Things.

Internet of Things technology develops through three social backgrounds: IT technology and industry, management science, and economic and social development. IT technology and industry realize Internet of Things technology through seven aspects: automation, computer, and communication. Under the background of the development of management science, it is realized through e-commerce and modern logistics. Under the background of economic and social development, it is realized through industrial structure transformation, industrialization and information integration, production internationalization, and so on.

Under this development background, IOT technology is gradually applied to smart grid, digital ocean, satellite positioning, and other industries, especially in power system. Various key technologies of IOT technology will be used in IOT architecture. Its application technology is shown in Figure 2.

As can be seen from the figure, there are many key technologies in the Internet of Things technology, such as cloud computing technology, network communication technology, sensor technology, and other key technologies.

\subsection{Intelligent Electrical Equipment Monitoring and Early} Warning System. Electrical equipment monitoring and early warning system is an important part of intelligent energy system and an important embodiment of intelligent energy system. By introducing a variety of advanced technologies such as Internet of Things technology, modern communication technology, visualization technology, and control technology, the system intuitively shows the operation safety of electrical equipment such as generators, the intelligent monitoring, and early warning of transformers and power lines in the energy system, as well as substations and power, so as to realize the integration with ERP infrastructure management and control module PMS information access, so as to realize the integrated automation system and provide important support for the safe operation, overhaul, and maintenance of the safety early warning system.

The intelligent system takes the power architecture as the foundation, integrates the Internet of Things technology, and constructs the three-tier structure of the substation: process layer, bay layer, and station control layer. Data transmission through wireless communication provides support for intelligent power informatization, automation, and interaction. Figure 3 shows the overall structure of the intelligent control system for electrical equipment.

In the three-tier structure, the properties are different: the bay layer mainly refers to relay protection, measurement and control, wave recording, etc.; the station control layer mainly refers to plant level monitoring, and the process layer 


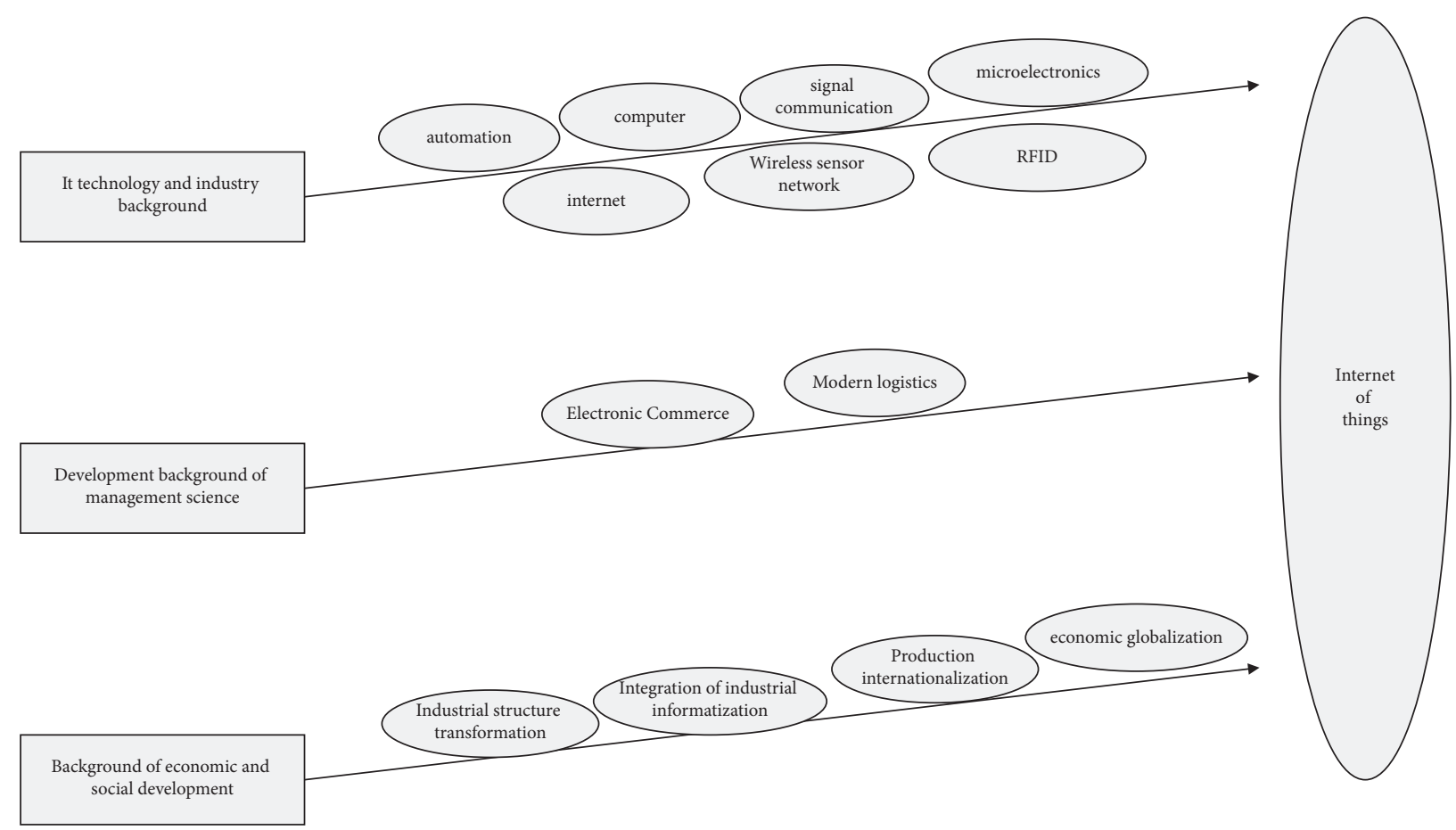

Figure 1: Development background of Internet of Things.

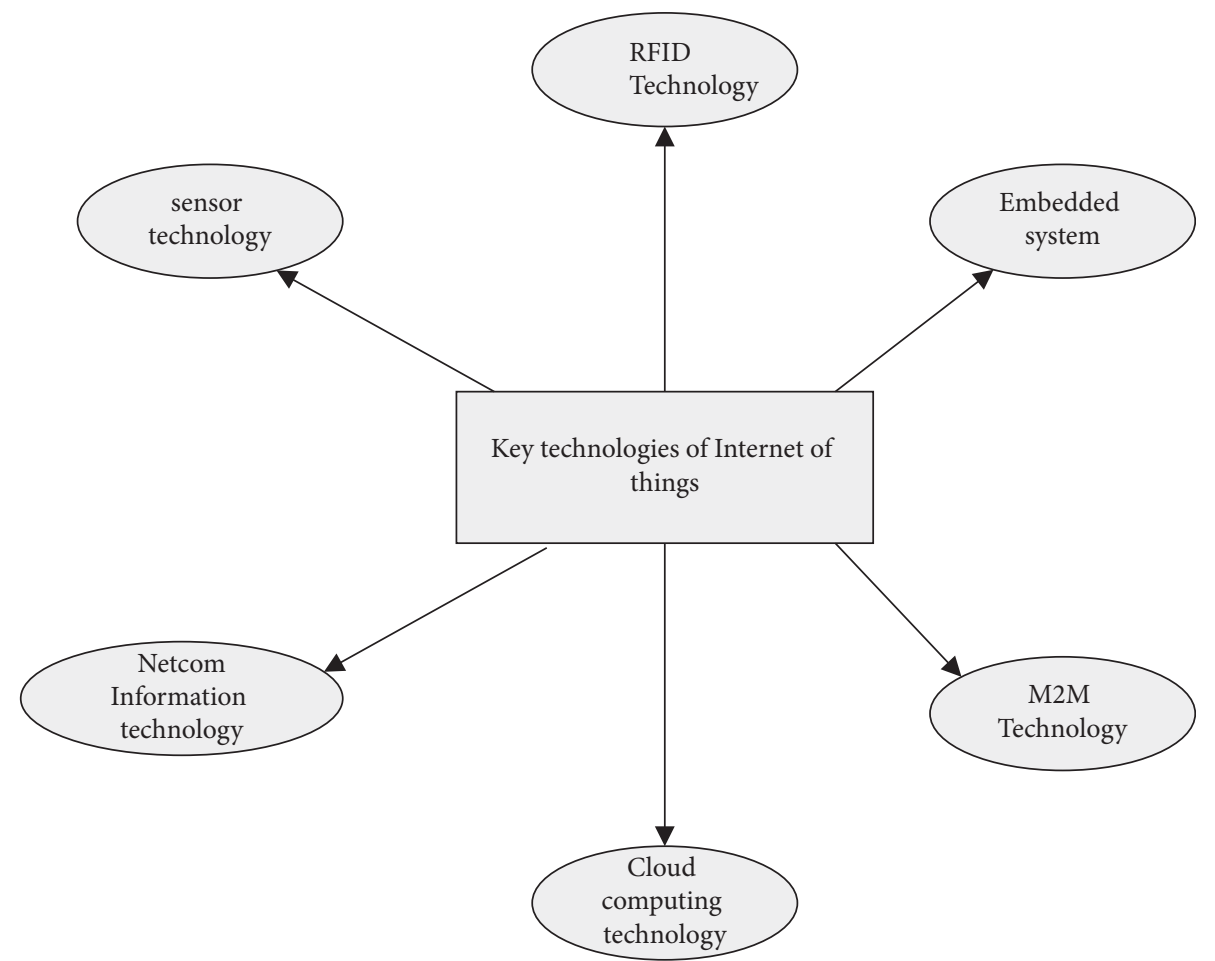

FIGURE 2: Key technologies of Internet of Things.

is the intelligent equipment in the exponential substation. The equipment at each level is different. The equipment at the station control level mainly refers to the monitoring master station, engineer station, information substation, etc. Process layer equipment includes intelligent circuit breaker replacement equipment, intelligent terminal, and electronic transformer merging unit.

Based on the analysis of the hierarchical structure model of the intelligent power system control system combining modern power system and Internet of Things technology, 


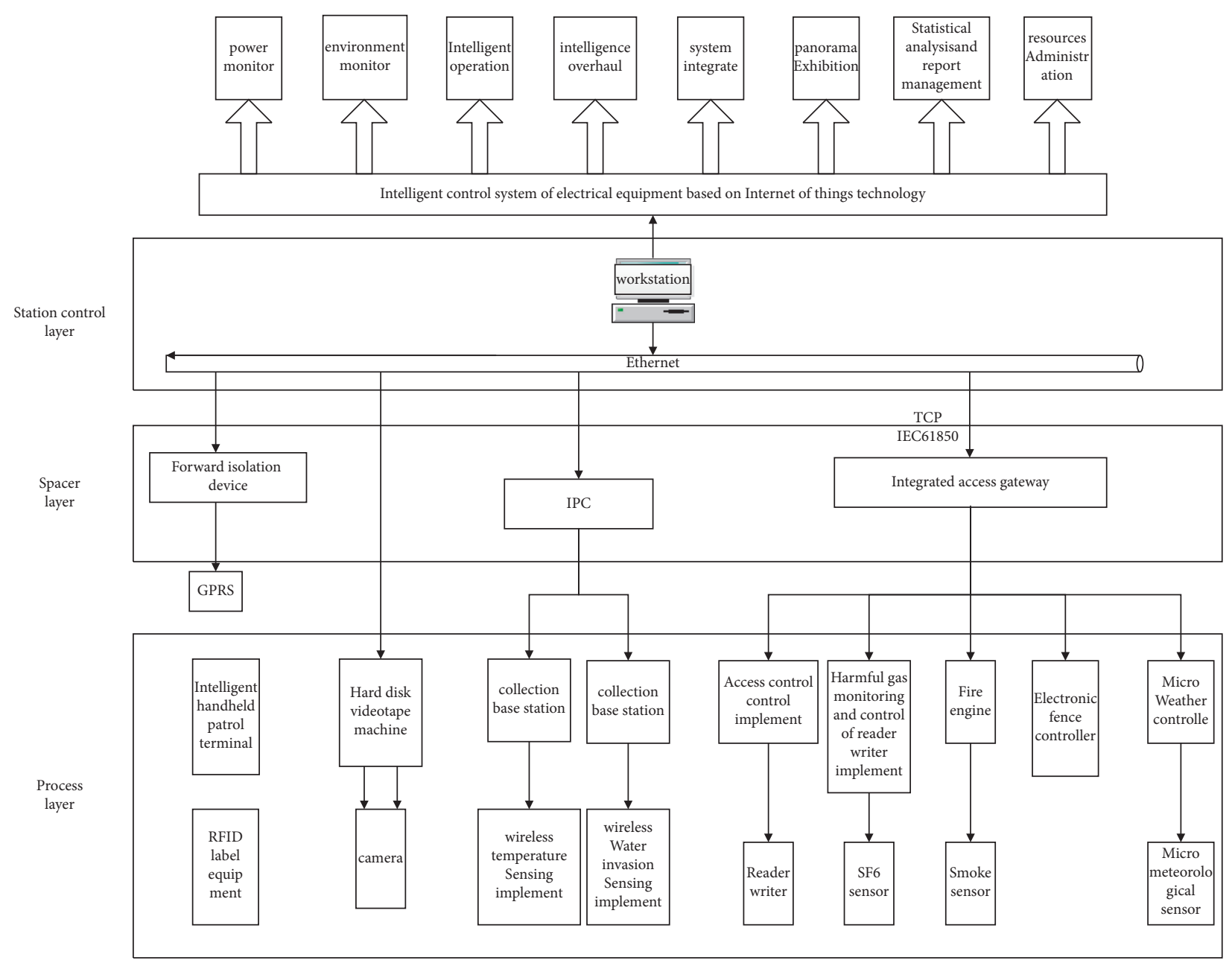

FIGURE 3: Overall structure of the intelligent control system for electrical equipment.

the process level of this system is like the state perception level of Internet of Things, and this level can best reflect the core technology of Internet of Things. This hierarchical structure collects the status signals of power equipment by means of wireless temperature sensor, wireless water intrusion sensor, RFID sensor, SF6 sensor, smoke sensor, and micro meteorological sensor and transmits the status data by means of Ethernet, wireless communication, and RS-485 bus. The second layer is the basic architecture connecting the station control layer and the process layer, which is equal to the network level in the Internet of Things. It can carry out information transmission such as online information monitoring data collection and control. All subsystems are interconnected through IEC61850 protocol. Finally, the station control layer is also equal to the application layer of the Internet of Things to control the whole system. The system workstation realizes the unified reception and analysis of signals for early warning and visual display. Unidirectional isolation equipment and integrated automatic control system can also be used to achieve the effect of big data interconnection.

Each subsystem in the whole system has different divisions of labor. According to different business functions, it is divided into three functional modules: power monitoring, environmental monitoring, and operation and maintenance. The main goal of the power monitoring module is to monitor the operation energy of intelligent primary and secondary equipment. It mainly collects the data of the substation integrated automation system for visual display, alarm threshold setting, and camera linkage. It realizes the real-time monitoring of battery voltage, current, and operation status. The environmental monitoring module monitors the buildings around the substation and the changes of the surrounding environment, including security monitoring, operation environment monitoring, and indoor intelligent management. The safe operation and maintenance of electrical equipment is the basic work to ensure the substation. The module automatically collects the RFID tag information of electrical equipment for intelligent inspection.

2.3. Collaboration between Cloud Computing and Edge Computing. Cloud computing can be understood as a kind of distributed computing. Through the network "cloud," a large number of data computing and processing programs are decomposed into countless small processing programs, which are analyzed and processed through a system 
composed of multiple servers. Cloud computing has the characteristics of large-scale, distributed, virtualization, high availability, high scalability, and more economic and security. Figure 4 reflects the Internet of Things architecture based on cloud computing.

The network "cloud" connects users and sensing devices, receives data from sensing devices, calculates, and stores the received data of electrical equipment operation in cloud computing mode and finally transmits the structure of analysis and processing to terminal devices.

Edge computing is a computing mode for computing, processing, and analyzing at the edge of the network. An edge can be any resource between a data access point and a cloud. The principle of edge computing is to calculate and process in the accessories of the data access point, realize intelligence at the network edge, and provide intelligent services nearby.

2.4. Security Monitoring Based on Wireless Sensor Network (WSN) Technology. Wireless sensor network technology is a network structure composed of a large number of intelligent sensor nodes deployed in the monitoring area. This structure integrates sensor technology, embedded computing, distributed information processing technology, and wireless communication technology for real-time monitoring, information perception and collection, data processing and analysis, and transmission to the terminal. The system based on wireless sensor technology has the characteristics of large capacity, low wiring cost, not affected by harsh environmental conditions and high monitoring accuracy. It is widely used in the safety early warning system of electrical equipment. It has strong ability to interfere with the environment and can realize wireless mode, which greatly reduces the space occupancy and system complexity. It has great advantages and functions in the power system, and its structure is shown in Figure 5.

Sensor nodes are distributed in the whole monitoring system to form a wireless network in the form of organization, transmit the collected data to the base station, analyze and process the data, then use it evenly on the Internet, transmit the mobile network or satellite to the pocket node, and finally configure it to manage and control the sensor network through the task node.

2.5. Safety Prediction of the System Based on Regression Analysis. The so-called safety prediction of electrical equipment is to predict the data of operation status through scientific methods such as Internet of Things technology and judge the place and situation of possible failure, including cause, process, and severity. Through the prediction of dangerous conditions, early warning, and corresponding safety measures for their occurrence, we can avoid risks, ensure safety, and save resources.

Regression analysis is to determine the relationship between independent variables and dependent variables according to the principle of mathematical statistics, establish a linear regression equation, and calculate the change trend of dependent variables through the equation.
According to the model, the regression equation is determined according to the previous electrical equipment fault data, and the development trend of error is predicted.

Gauss Markov hypothesis: the vector composed of random variables has normal characteristics.

Mathematical expectation:

$$
E(\varepsilon)=0 .
$$

Variance covariance matrix:

$$
\begin{aligned}
E\left(\mathcal{\varepsilon}^{\prime 2}\right) & =\sigma^{2} I \triangle \operatorname{Cov}(\varepsilon), \\
\varepsilon & \sim N\left(0, \sigma^{2} I\right) .
\end{aligned}
$$

The expected value of the random vector $y$ condition obtained based on formula (1):

$$
E(Y \mid X)=X B .
$$

Variance covariance matrix:

$$
\begin{aligned}
\operatorname{Cov}(Y) & =\sigma^{2} I, \\
x_{j}, j & =1,2,3, \ldots, k .
\end{aligned}
$$

Here, $x_{j}$ is a definite variable, there is no random component, and there is no correlation between its element components. Based on formula (1), the least-squares estimators of $b_{0}, b_{1}, \ldots, b_{k}$, where the sampling distribution obeys the positive distribution:

$$
E\left(b_{i}\right)=B_{i},
$$

where $b_{i}$ is an unbiased estimate of $B_{i}$; i.e., the variance is

$$
\operatorname{Var}\left(b_{i}\right)=\frac{\sigma^{2}}{1-R_{i}^{2}} .
$$

Among them, $R_{i}^{2}$ is the negative correlation coefficient, and then the normal equation is established.

$K$ factors are known in multiple linear regression, that is, $k$-ary linear regression. Sample data are

$$
\left\{\begin{array}{l}
x_{11}, x_{21}, \ldots, x_{k 1}, y_{1} \\
x_{12}, x_{22}, \ldots, x_{k 2}, y_{2}, \\
., \\
. \\
x_{1 i}, x_{2 i}, \ldots, x_{k i}, y_{i}, \\
., \\
, \\
. \\
x_{1 n}, x_{2 n}, \ldots, x_{k n}, y_{n} .
\end{array}\right.
$$

To establish the regression model:

$$
y_{i}^{*}=b_{0}+\sum_{i=1}^{k} b_{i} x_{i t} .
$$




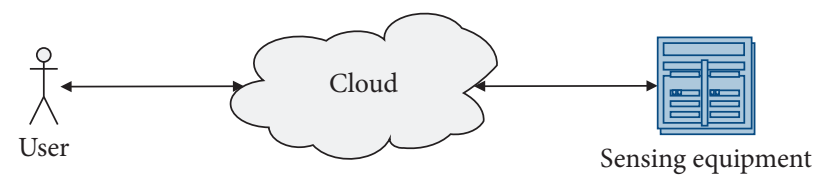

Figure 4: Data flow of cloud computing.

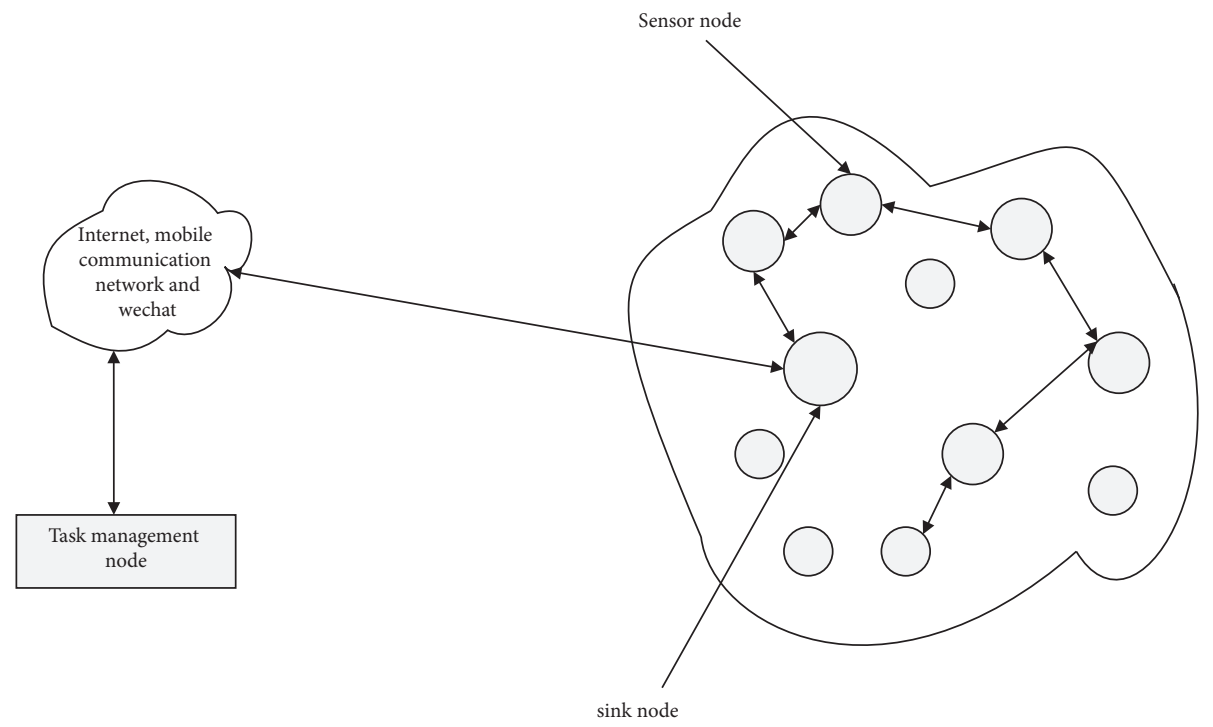

Figure 5: Wireless sensor network architecture.

According to the least-squares method, $b_{i}(i=1,2, \ldots, k)$ normal equation:

$$
\begin{aligned}
& \left(\begin{array}{cccc}
S_{11} & S_{12} & \cdots & S_{1 k} \\
S_{21} & S_{22} & \cdots & S_{2 k} \\
\vdots & \vdots & \vdots & \vdots \\
S_{k 1} & S_{k 2} & \cdots & S_{1 k k}
\end{array}\right)\left(\begin{array}{c}
b_{1} \\
b_{2} \\
\vdots \\
b_{k}
\end{array}\right)=\left(\begin{array}{c}
S_{1 k} \\
S_{2 y} \\
\vdots \\
S_{k y}
\end{array}\right) \\
& \left\{\begin{array}{c}
S_{i j}=S_{j i}=\sum_{t=1}^{n}\left(x_{i t}-\overline{x_{i}}\right)\left(x_{i j}-\overline{x_{j}}\right) \leq X_{i}-\overline{x_{i}}, X_{j}-\overline{x_{j}}> \\
S_{i i}=\sum_{t=1}^{n}\left(x_{i t}-\overline{x_{i}}\right)^{2} \leq X_{i}-\overline{x_{i}}, X_{i}-\overline{x_{i}}> \\
S_{i y}=\sum_{t=1}^{n}\left(x_{i t}-\overline{x_{i}}\right)\left(y_{i}-\bar{y}\right) \leq X_{i}-\overline{x_{i}}, Y_{i}-\overline{y_{i}}> \\
\bar{y}=\sum_{t=1}^{n} y_{t / n}, \overline{x_{i}}=\sum_{t=1}^{n}\left(\frac{x_{i t}}{n}\right) \\
j=1,2, \ldots, k .
\end{array}\right.
\end{aligned}
$$$$
S_{i j}=S_{j i} \text {, Therefore, we can obtain }
$$

$$
\left(S_{i j}\right)=\left(\begin{array}{cccc}
S_{11} & S_{12} & \cdots & S_{1 k} \\
S_{21} & S_{22} & \cdots & S_{2 k} \\
\vdots & \vdots & \vdots & \vdots \\
S_{k 1} & S_{k 2} & \cdots & S_{1 k k}
\end{array}\right) .
$$

Simplifying $(b)$ and $\left(S_{y}\right)$ :

$$
\begin{aligned}
& (b)=\left(\begin{array}{c}
b_{1} \\
b_{2} \\
\vdots \\
b_{k}
\end{array}\right), \\
& \left(S_{y}\right)=\left(\begin{array}{c}
S_{1 k} \\
S_{2 y} \\
\vdots \\
S_{k y}
\end{array}\right),
\end{aligned}
$$

we can obtain

$$
\left(S_{i j}\right)(b)=\left(S_{y}\right) \text {. }
$$

$\left(S_{i j}\right)$ If it is reversible, the following solution can be obtained:

$$
(b)=\left(S_{i j}\right)^{-1}\left(S_{y}\right) .
$$

So, we can obtain 


$$
b_{0}=\bar{y}-\sum_{i=1}^{k} b_{i} \overline{x_{i}}
$$

Finally, the regression equation is obtained:

$$
\widehat{y}=b_{0}+\sum_{i=1}^{k} b_{i} x_{i} \text {. }
$$

\section{Security Early Warning Based on Various Algorithms}

3.1. Kalman Filtering Algorithm. The algorithm estimates the real-time data and the corresponding state of the previous moment and recurses through the system state transition equation. For any dynamic system, Kalman filtering model can be divided into state and observation equations:

$$
\begin{aligned}
X_{k} & =F_{(k / k-1)} X_{k-1}+G_{k-1} W_{k-1}, \\
L_{k} & =H_{k} X_{k}+V_{k},
\end{aligned}
$$

where $X_{k}$ represents the n-1 order state vector at time $k, L_{k}$ represents the m-1 order state vector at time $k, G_{k-1}$ represents the system time $n \times r$ dynamic noise matrix of order $r$, $F_{(k / k-1)}$ indicates that the system changes from time k-1 to time $k n \times n$ order state transition matrix, $V_{k}$ represents the observation noise of order m-1 at time $k$ of the system, and $H_{k}$ represents system $\mathrm{K}$ time $m \times n$ order observation matrix. Setting $W_{k}$ as the dynamic noise and $V_{k}$ as the observation noise, which is a Gaussian white noise sequence, their statistical characteristics are

$$
\begin{aligned}
& \left\{\begin{array}{l}
E\left[W_{k}\right]=0, \\
E\left[V_{k}\right]=0,
\end{array}\right. \\
& \left\{\begin{array}{l}
E\left[W_{k} W_{j}^{T}\right]=Q_{k} \delta_{k j}, \\
E\left[V_{k} V_{j}^{T}\right]=R_{k} \delta_{k j},
\end{array}\right.
\end{aligned}
$$

where $Q_{k}$ and $R_{k}$ are the variance matrix of dynamic noise and observation noise and $\delta_{k j}$ is the logic function.

$$
\delta_{k j}=\left\{\begin{array}{l}
0,(k \neq j), \\
1,(k=j) .
\end{array}\right.
$$

$W_{k}, V_{k}$ irrelevant:

$$
\begin{aligned}
& E\left[W_{k} V_{j}^{T}\right]=0, \\
& \left\{\begin{array}{l}
\widehat{X_{0}}=E\left(X_{0}\right)=\mu x_{0}, \\
\widehat{P}_{0}=\operatorname{Var}\left(X_{0}\right) .
\end{array}\right.
\end{aligned}
$$

$X_{0}$ is the initial state of the system, normal distribution, and $W_{k}, V_{k}$ irrelevant:

$$
\left\{\begin{array}{l}
E\left[X_{0} W_{j}^{T}\right]=0, \\
E\left[X_{0} V_{j}^{T}\right]=0 .
\end{array}\right.
$$

Set target initial state $X_{0}, P_{0}$ to get the least square difference of $X_{t}$.
Calculate the residual first:

$$
\widetilde{Z}_{(k / k-1)}=Z_{k}-H(k) \widehat{X}_{(k / k-1)} .
$$

Then calculate the residual covariance:

$$
\begin{aligned}
S_{(k / k-1)} & =E\left[\widetilde{Z}_{(k / k-1)} \widetilde{Z}_{(k / k-1)}^{r}\right] \\
& =H(k) P_{(k / k-1)} H(k)^{r}+R_{k} .
\end{aligned}
$$

Calculate the gain:

$$
K_{(k / k)}=P_{(k / k-1)} H_{k}^{T} H_{(k / k-1)}^{T} .
$$

Finally, update the status:

$$
\widehat{X}_{k}=\widehat{X}_{(k / k-1)}+K_{k}\left(Z_{k}-H_{k} \widehat{X}_{(k / k-1)}\right) \text {. }
$$

Therefore, the Kalman filtering algorithm is executed recursively after sampling in each cycle.

3.2. BP Neural Network Algorithm. BP network can also be called backpropagation neural network. Its structure can be simply divided into three layers: feedforward, multilayer, and perceptron network. This algorithm repeatedly trains the collected sample data and constantly modifies the network weight and threshold to minimize the error and achieve the desired goal for the output data. BP generally includes input layer middle layer (hidden layer), and output layer. Each layer consists of several neuron groups and is fully connected with the previous layer by switching weights. In the input layer, when an input neuron receives a signal, it is transmitted to each neuron in the middle layer (hidden layer) (hidden layer), each neuron calculates the sum, and then the nonlinear activation function is used to generate the output signal and transmit it to the output layer. The processing of the last message transmitted to the output stage, that is, the subsequent upward increase, is considered to have been completed, and the starting layer is responsible for displaying the results to the outside world. In case of actual loss, if the output does not match the expected output, it is necessary to perform the error return multiplication procedure. Output error starts from the output, adjusts and updates the weight and threshold of each layer, and gradually converts the transmission to the middle layer and input layer. The process of repeated information forward expansion and error recovery is to adjust the weight of each layer of the whole neural network.

In the calculation process, only when the set training times or the calculated global network error is less than the general value, if the global error accuracy error is very small, the whole learning process of the algorithm ends. If you are not satisfied with the results, you must update the training mode. A round of learning process runs again until the final conditions are met, and the BP three-layer network model can meet the requirements of high precision.

3.3. Kalman-BP Neural Network Algorithm. Kalman algorithm and BP algorithm have their own advantages. They are widely used in many fields such as Internet of Things 
technology and have very high practical value. However, there are still some deficiencies in the application of these two algorithms to the field of equipment safety early warning. For example, Kalman algorithm must be based on the existing accurate model and existing data for calculation. For large and complex systems, the establishment of accurate model and data acquisition are not so easy, and the measurement accuracy cannot be realized. BP algorithm can meet the needs of high precision, but it has some problems, such as small part, slow speed, and weak ability to external interference. Therefore, we can combine the two to form a new algorithm, namely, Kalman-BP neural network algorithm.

This algorithm can combine the two algorithms to make up for the shortcomings of their algorithms and achieve the desired effect. This algorithm replaces the characteristics of adjusting and updating weights and thresholds in BP algorithm with Kalman filtering algorithm and uses Kalman algorithm to train adjusting and updating weights and thresholds. The filtering idea is mainly brought into the BP audit network and optimized after adjustment; that is, the mathematical model is

$$
\left\{\begin{aligned}
W_{k+1} & =I_{k} W_{k}+\omega_{k}, \\
y_{k+1} & =H_{k+1}\left(W_{k+1} U_{k+1}\right),
\end{aligned}\right.
$$

where $W_{k}$ is the BP weight vector; $\omega_{k}$ is the system process noise; $I_{k}$ is the state transition matrix; $y_{k}$ is the expected output value; and $H_{k}$ is the observation matrix.

\section{Experimental Comparison and Analysis}

We collected real data and compared three algorithms, Kalman algorithm, BP algorithm, and Kalman BP algorithm, taking the lightning protection safety facility SPD (surge protector) in electrical equipment as an example, the voltage and current state data of electrical equipment are verified. The voltage of surge protector is mainly affected by electric field voltage, current and temperature. The input data of three algorithms are compared and analyzed, respectively, to obtain the optimal algorithm.

4.1. For Prediction Error Value. 12 groups of data are extracted from the data collected continuously for 45 days and 1080 hours for experimental analysis. The predicted values are compared with the measured values by using Kalman algorithm, BP algorithm, and Kalman BP algorithm, and the error value is obtained. The optimal algorithm is the one with the smallest error, as shown in Table 1.

Comparison of 12 groups of data under phase A voltage and their relative error values are shown in Figures 6 and 7:

It can be seen from the comparison diagram of the two experimental results that under the A-phase voltage, the Kalman-BP algorithm, a combination of BP algorithm and Kalman algorithm, is much better than the original single algorithm. The error value of this algorithm is less than $4 \%$, and neither Kalman algorithm nor BP algorithm can achieve this effect. For the accuracy of the results, the comparison of error values under phase $\mathrm{B}$ voltage and phase C voltage is also carried out, as shown in Figures 8 and 9.

Through comparative experiments, for phase B and C voltages, the relative error of the prediction results of Kalman BP algorithm is still less than that of the traditional algorithm. Therefore, the function of Kalman-BP algorithm for electrical equipment safety early warning system is greater than that of the traditional algorithm. It can be closer to the actual prediction value and achieve better prediction effect. It can be used for monitoring and prediction of surge protector and early warning of equipment operation state, which has great practical value.

4.2. Calculation Speed. Finally, we also compare the operation speeds of the three algorithms under three voltages and calculate them in milliseconds (MS), as shown in Figures 10-12.

Through the experimental data, it is found that the speed of Kalman BP algorithm is obviously better than the other two algorithms. This algorithm has obvious advantages under any voltage. It is also more helpful in practical application.

After the whole experiment, through the experimental data, it can be seen that this system realizes relevant intelligent application services, which can ensure not only the stability of information transportation but also real-time monitoring of electrical equipment, early warning, shorten the troubleshooting time, reduce the workload of power station staff, and achieve the role of safety early warning, emergency command, and control. It is of great significance to the monitoring and early warning system of electrical equipment.

4.3. Experimental Summary. Through the comparison of several algorithms in the above experiments, it is not difficult to see that Kalman-BP algorithm is obviously superior to Kalman and BP algorithm in terms of computational efficiency and accuracy. This algorithm can verify the results more accurately under phase A, B, and C voltages. Therefore, the algorithm based on Internet of Things technology for monitoring and early warning of electrical equipment is very important. This algorithm can reduce the amount of data calculation, improve efficiency, remove redundancy, and improve calculation accuracy. This system realizes relevant intelligent application services, which can not only ensure the stability of information transportation but also monitor the electrical equipment in real time, give early warning in advance, shorten the troubleshooting time, reduce the workload of power station staff, and achieve the role of safety early warning, emergency command and control. It is of great significance to the monitoring and early warning system of electrical equipment.

In the electrical equipment monitoring and early warning system, the Internet of Things technology must become the main research and development of today's society. Because in modern society, Internet technology basically occupies the whole life of human production and life, which is basically inseparable from the participation of the 
TABLE 1: Comparison between measured voltage and predicted voltage.

\begin{tabular}{|c|c|c|c|c|c|c|c|}
\hline \multirow{2}{*}{$\begin{array}{l}\text { Serial number } \\
\text { Time }\end{array}$} & \multirow{2}{*}{$\begin{array}{c}\text { Measured value } \\
\text { Company_V }\end{array}$} & \multicolumn{2}{|c|}{ Kalman algorithm } & \multicolumn{2}{|c|}{ BP algorithm } & \multicolumn{2}{|c|}{ Kalman-BP algorithm } \\
\hline & & Forecast & Error & Forecast & Error & Forecast & Error \\
\hline 1 & 224.0 & 220.3 & -3.7 & 221.3 & -2.7 & 223.0 & -1.0 \\
\hline 2 & 227.0 & 222.0 & -5 & 222.7 & -4.3 & 223.5 & -3.5 \\
\hline 3 & 227.1 & 221.2 & -5.9 & 223.2 & -3.9 & 224.0 & -3.1 \\
\hline 4 & 228.5 & 223.6 & -4.9 & 223.7 & -4.8 & 224.3 & -4.2 \\
\hline 5 & 224.1 & 226.1 & 2.0 & 225.9 & 1.8 & 224.8 & 0.7 \\
\hline 6 & 223.9 & 220.5 & -3.4 & 226.6 & 2.7 & 224.9 & 1.0 \\
\hline 7 & 227.8 & 221.1 & -6.7 & 225.1 & -2.7 & 225.7 & -2.1 \\
\hline 8 & 227.5 & 223.3 & -4.2 & 223.2 & -4.3 & 223.9 & -3.6 \\
\hline 9 & 226.5 & 221.4 & -5.1 & 220.2 & -6.3 & 222.0 & -4.5 \\
\hline 10 & 222.0 & 228.8 & 6.8 & 229.5 & 7.5 & 227.8 & 5.8 \\
\hline 11 & 222.2 & 230.1 & 7.9 & 229.6 & 7.4 & 227.5 & 5.3 \\
\hline 12 & 222.5 & 232.5 & 10 & 231.2 & 8.7 & 228.5 & 6.0 \\
\hline
\end{tabular}

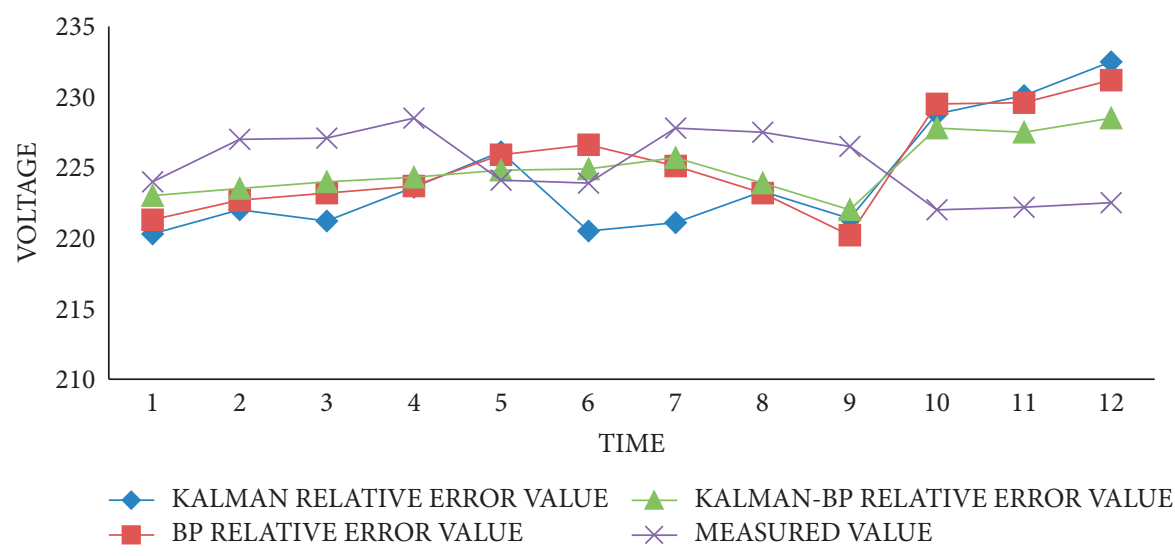

Figure 6: Comparison between predicted and measured phase A voltage.

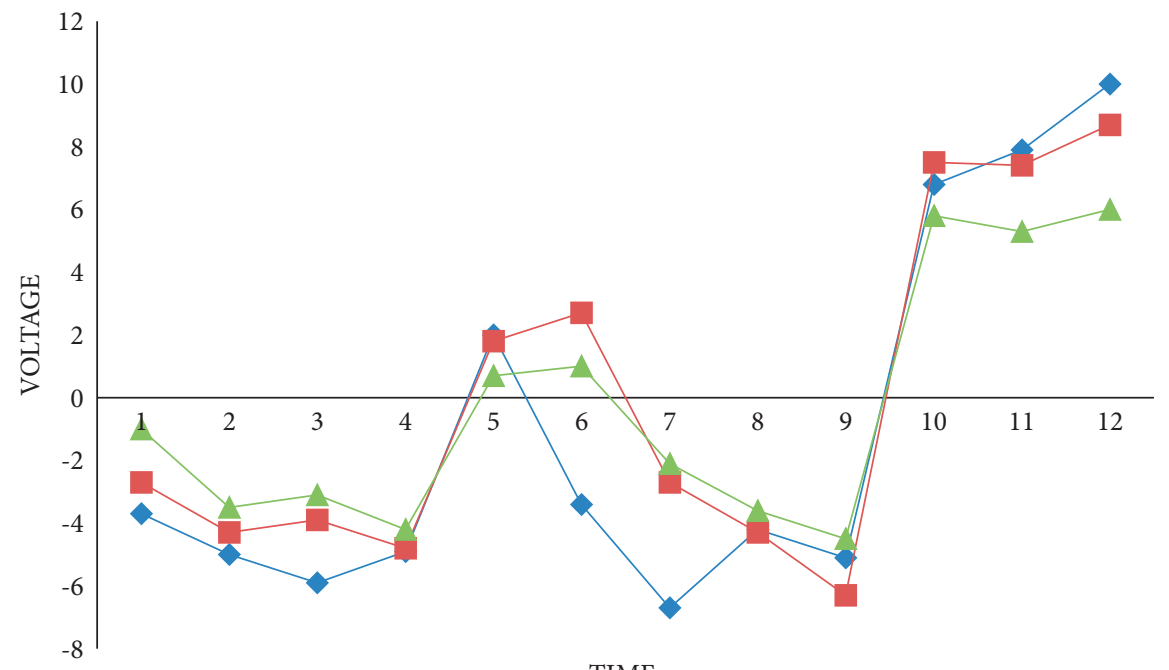

TIME

KALMAN RELATIVE ERROR VALUE

BP RELATIVE ERROR VALUE

- KALMAN-BP RELATIVE ERROR VALUE

Figure 7: Relative error of predicted value of phase A voltage. 


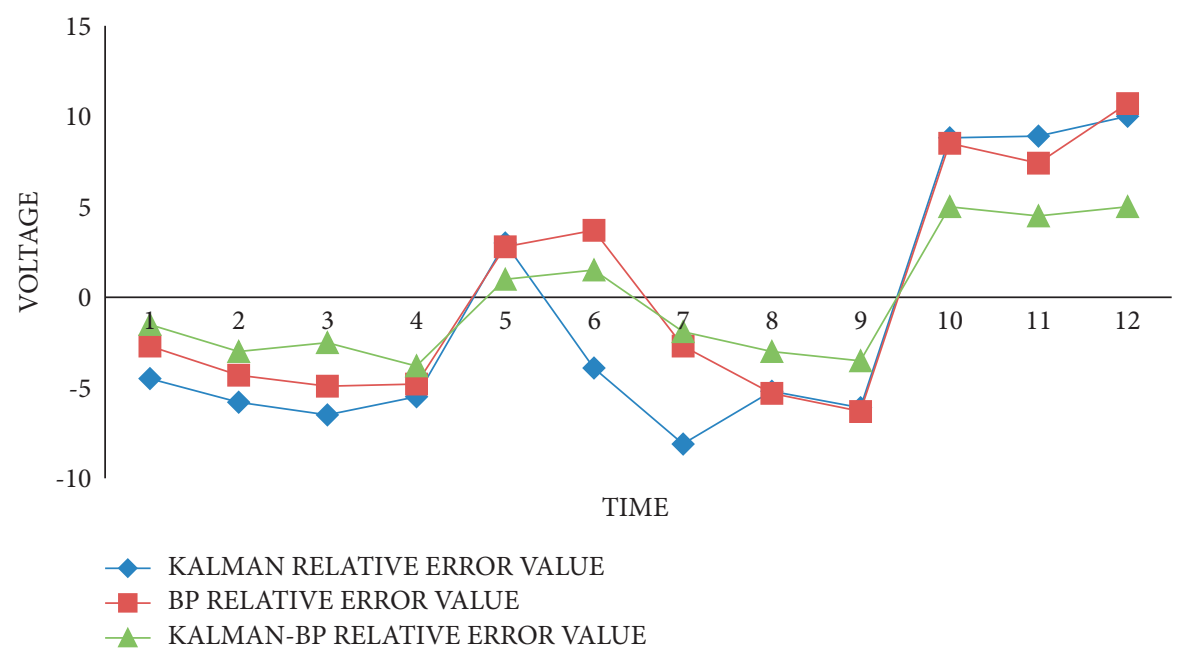

FIgURE 8: Relative error of phase B voltage prediction value.

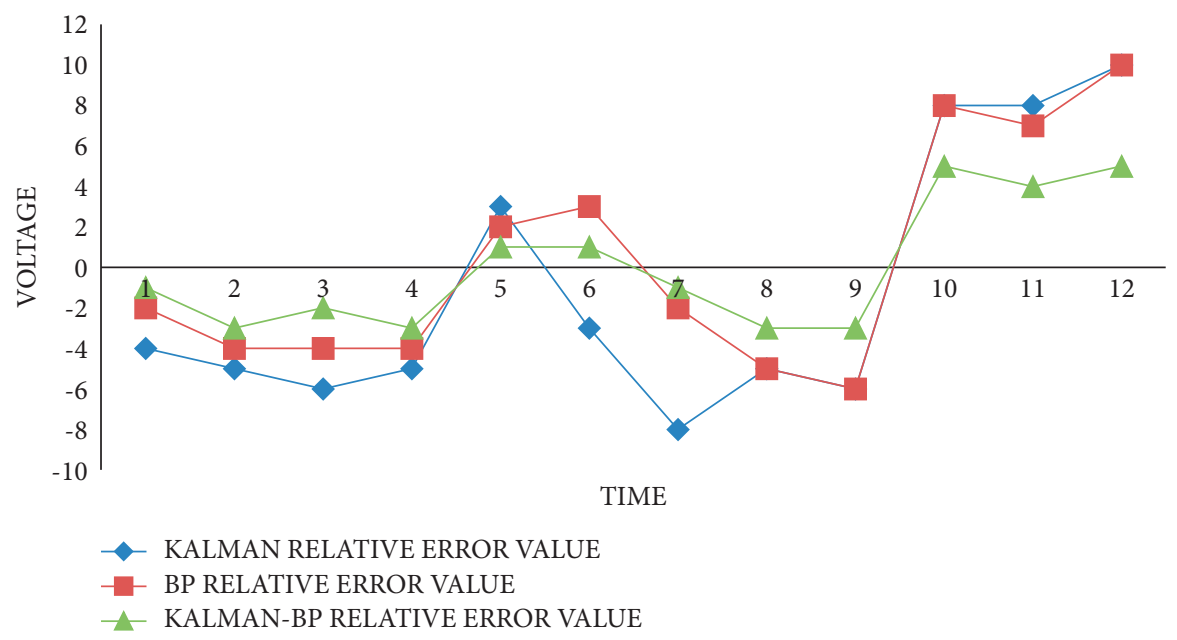

FIGURE 9: Relative error of predicted value of phase $C$ voltage.

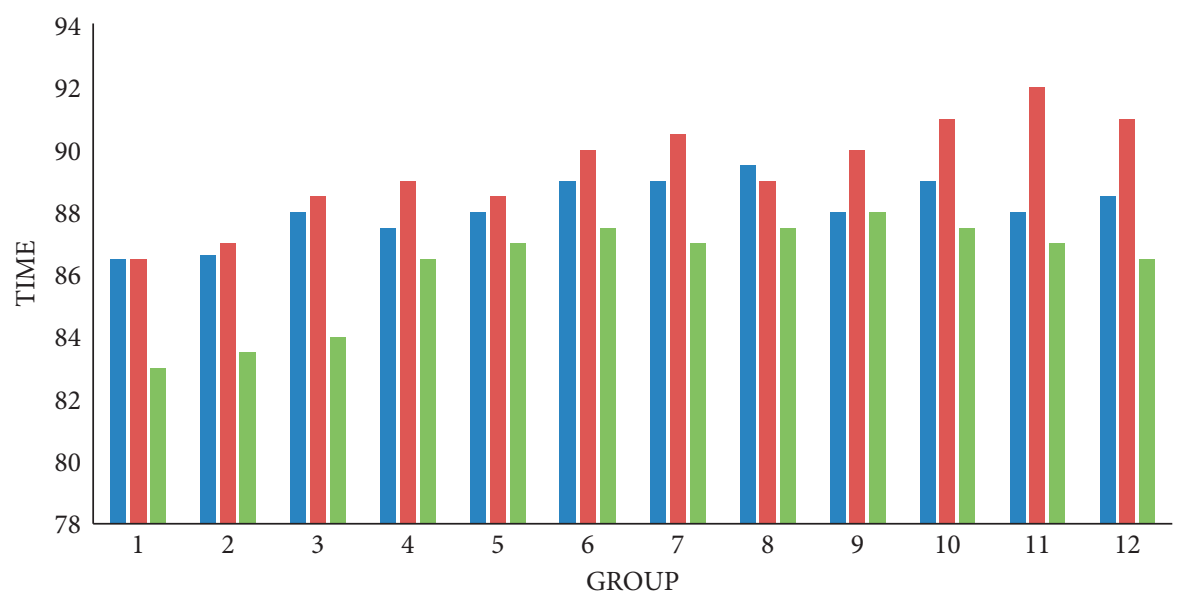

- KALMAN ALGORITHM

- BP ALGORITHM

- KALMAN-BP ALGORITHM

FIgURE 10: Operation speed under phase A voltage. 

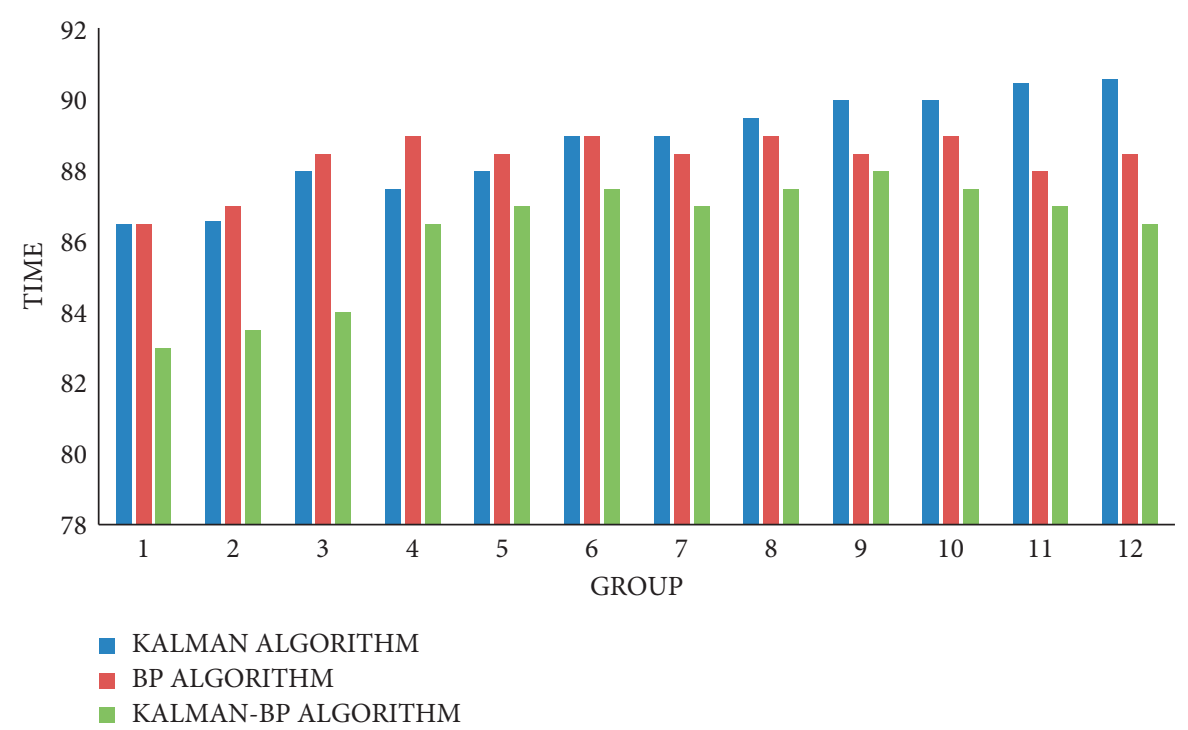

Figure 11: Operation speed under phase B voltage.

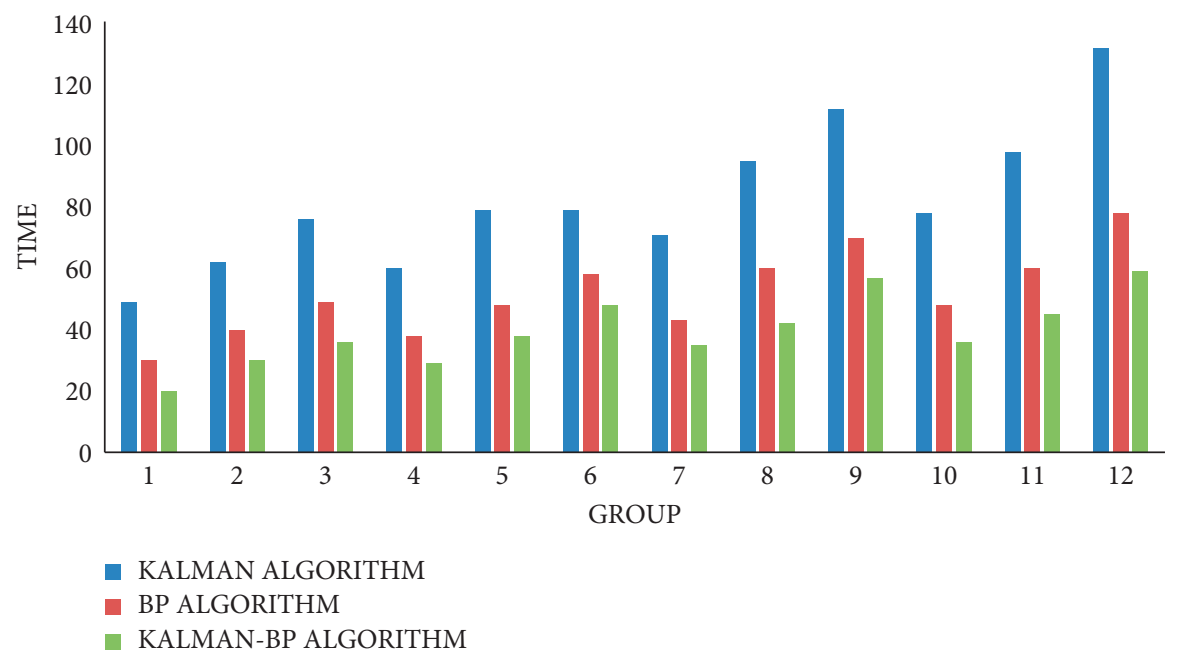

Figure 12: Operation speed under phase $\mathrm{C}$ voltage.

Internet. Therefore, the development and application of Internet of Things technology will bring great changes to our production and life.

\section{Conclusion}

At present, the monitoring and early warning system of electrical equipment is not perfect and is still in the early stage of construction. Technicians are also required to carry out experimental research and development. However, with the rapid development of modern Internet of Things technology, building large and complex systems with Internet of Things has become a basic trend. Therefore, the construction of monitoring and early warning system for electrical equipment through Internet of Things technology is helpful to monitor the change trend of risk factors in real time and improve the sensitivity and reference of risk factors. In the case of no one on duty, it effectively solves the problem of no monitoring of power equipment in the parts prone to safety accidents, so as to achieve online and realtime monitoring, prevent equipment damage caused by various factors, and improve the reliability and safety of operation.

Therefore, the development and application of Internet of Things technology will bring great changes to our production and life and bring new vitality to all fields of life. As an important industry for global development, power industry is particularly important for power energy economy. Through the research on power generation monitoring and early warning, we can find new and better methods to implement safety management and control, provide more opportunities to improve the internal safety of power grid, equipment, and personnel, improve performance, and improve people's happiness index. 


\section{Data Availability}

The experimental data used to support the findings of this study are available from the corresponding author upon request.

\section{Conflicts of Interest}

The authors declare that they have no conflicts of interest regarding this work.

\section{References}

[1] C. Lang and H. Utikal, "Organisatorische impulse durch internet-technologie und technologieinduzierte Strategien," E-Organisation, Gabler Verlag, German, UK, 2002.

[2] L. Yang, S. H. Yang, and L. Plotnick, "How the internet of things technology enhances emergency response operations," Technological Forecasting and Social Change, vol. 80, no. 9, pp. 1854-1867, 2013.

[3] R. R. Sepasi, A. Abbaszadeh, F. Borhani, and H. Rafiei, "Nurses' perceptions of the concept of power in nursing: a qualitative research," Journal of Clinical and Diagnostic Research, vol. 10, no. 12, pp. 10-15, 2016.

[4] J. Goldemberg, "The promise of clean energy," Energy Policy, vol. 34, no. 15, pp. 2185-2190, 2006.

[5] X. Fang, S. Misra, and G. Xue, "Smart grid-the new and improved power grid: a survey," IEEE communications surveys \& tutorials, vol. 14, no. 4, pp. 944-980, 2011.

[6] D. P. Kothari, "Power System optimization," in Proceedings of the 2012 2nd National Conference on Computational Intelligence and Signal Processing (CISP), pp. 18-21, IEEE, Guwahati, India, March 2012.

[7] F. Fei Tao, Y. Ying Zuo, L. Li Da Xu, and Z. Lin, "IoT-based intelligent perception and access of manufacturing resource toward cloud manufacturing," IEEE Transactions on Industrial Informatics, vol. 10, no. 2, pp. 1547-1557, 2014.

[8] A. Kaiser and T. Schreiber, "Information transfer in continuous processes," Physica D: Nonlinear Phenomena, vol. 166, no. 1-2, pp. 43-62, 2002.

[9] Y. Han and Y. H. Song, "Condition monitoring techniques for electrical equipment-a literature survey," IEEE Transactions on Power Delivery, vol. 18, no. 1, pp. 4-13, 2003.

[10] Y. Yan and J. A. Malen, "Periodic heating amplifies the efficiency of thermoelectric energy conversion," Energy \& Environmental Science, vol. 6, no. 4, pp. 1267-1273, 2013.

[11] R. Katz, J. S. Karliner, and R. Resnik, "Effects of a natural volume overload state (pregnancy) on left ventricular performance in normal human subjects," Circulation, vol. 58, no. 3, pp. 434-441, 1978.

[12] M.-F. Harkat, G. Mourot, and J. Ragot, "An improved PCA scheme for sensor FDI: application to an air quality monitoring network," Journal of Process Control, vol. 16, no. 6, pp. 625-634, 2006.

[13] A. Challoner and G. H. Popescu, "Intelligent sensing technology, smart healthcare services, and internet of medical things-based diagnosis," American Journal of Medical Research, vol. 6, no. 1, pp. 13-18, 2019.

[14] L. Jin, W. Zhang, H. Zhang et al., "An embedded FBG sensor for simultaneous measurement of stress and temperature," IEEE Photonics Technology Letters, vol. 18, no. 1, pp. 154-156, 2005.

[15] H. S. Tzou and C. I. Tseng, "Distributed piezoelectric sensor/ actuator design for dynamic measurement/control of distributed parameter systems: a piezoelectric finite element approach," Journal of Sound and Vibration, vol. 138, no. 1, pp. 17-34, 1990.

[16] A.-s. Oh, "Smart factory logistics management system using house interior position tracking technology based on bluetooth beacon," Journal of the Korea Institute of Information and Communication Engineering, vol. 19, no. 11, pp. 26772682, 2015.

[17] R. Want, "An introduction to RFID technology," IEEE pervasive computing, vol. 5 , no. 1, pp. 25-33, 2006.

[18] M. Franceschinis, M. A. Spirito, R. Tomasi, G. Ossini, and M. Pidalà, "Using wsn technology for industrial monitoring: a real case," in Proceedings of the 2008 Second International Conference on Sensor Technologies and Applications (Sensorcomm 2008), pp. 282-287, IEEE, Cap Esterel, France, August 2008.

[19] Z. Ujhelyi, G. Bergmann, A. Hegedues et al., "EMF-IncQuery: an integrated development environment for live model queries," Science of Computer Programming, vol. 98, pp. 80-99, 2015.

[20] M. El Arass, I. Tikito, and N. Souissi, "data lifecycles analysis: towards intelligent cycle," in Proceedings of the 2017 Intelligent Systems and Computer Vision (ISCV), pp. 1-8, IEEE, Fez, Morocco, April 2017.

[21] L. Pengfei, L. Jiakun, and J. Junfeng, "Wireless temperature monitoring system based on the ZigBee technology,"vol. 1, pp. V1-V160-V1-163, in Proceedings of the 2010 2nd international conference on computer engineering and technology, vol. 1, IEEE, Chengdu, China, April 2010.

[22] R. Usamentiaga, P. Venegas, J. Guerediaga, L. Vega, J. Molleda, and F. Bulnes, "Infrared thermography for temperature measurement and non-destructive testing," Sensors, vol. 14, no. 7, Article ID 12305, 2014.

[23] C. Maier, "Infrared temperature measurement of polymers," Polymer Engineering \& Science, vol. 36, no. 11, pp. 1502-1512, 1996.

[24] G. Chen, L. Wang, and M. M. Kamruzzaman, "Spectral classification of ecological spatial polarization SAR image based on target decomposition algorithm and machine learning," Neural Computing \& Applications, vol. 32, no. 10, pp. 5449-5460, 2020. 